\title{
Aspirin for Primary Prevention of Cardiovascular Events
}

Federico A. Augustovski, MD, Scott B. Cantor, PhD, Chau T. Thach, BA, Stephen J. Spann, MD

\begin{abstract}
OBJECTIVE: The use of aspirin for primary prevention of cardiovascular events in the general population is controversial. The purpose of this study was to create a versatile model to evaluate the effects of aspirin in the primary prevention of cardiovascular events in patients with different risk profiles.
\end{abstract}

DESIGN: A Markov decision-analytic model evaluated the expected length and quality of life for the cohort's next 10 years as measured by quality-adjusted survival for the options of taking or not taking aspirin.

SETTING: Hypothetical model of patients in a primary care setting.

PATIENTS: Several cohorts of patients with a range of risk profiles typically seen in a primary care setting were considered. Risk factors considered included gender, age, cholesterol levels, systolic blood pressure, smoking status, diabetes, and presence of left ventricular hypertrophy. The cohorts were followed for 10 years. Outcomes were myocardial infarction, stroke, gastrointestinal bleed, ulcer, and death.

MAIN RESULTS: For the cases considered, the effects of aspirin varied according to the cohort's risk profile. By taking aspirin, the lowest-risk cohort would be the most harmed with a loss of 1.8 quality-adjusted life days by taking aspirin; the highest risk cohort would achieve the most benefit with a gain of 11.3 quality-adjusted life days. Results without quality adjustment favored taking aspirin in all the cohorts, with a gain of 0.73 to 8.04 days. The decision was extremely sensitive to variations in the utility of taking aspirin and to aspirin's effects on cardiovascular mortality. The model was robust to other probability and utility changes within reasonable parameters.

CONCLUSIONS: The decision of whether to take aspirin as primary prevention for cardiovascular events depends on patient risk. It is a harmful intervention for patients with no risk factors, and it is beneficial in moderate and high-risk patients. The benefits of aspirin in this population are comparable to those of other widely accepted preventive strategies. It is especially dependent on the patient's risk profile, patient prefer-

Received from the Hospital Italiano de Buenos Aires, Unidad de Medicina Familiar y Preventiva, Buenos Aires, Argentina (FAA); The University of Texas M. D. Anderson Cancer Center, Department of Medical Specialties, Section of General Internal Medicine, Houston, Tex. (SBC); The University of Washington, Department of Biostatistics, Seattle, Wash. (CTT); and Baylor College of Medicine, Department of Family and Community Medicine, Houston, Tex. (SJS).

Presented in part at the 18th annual meeting of the Society of General Internal Medicine, San Diego, Calif., May 1995.

Address correspondence and reprint requests to Dr. Cantor: The University of Texas, M.D. Anderson Cancer Center, Department of Medical Specialties, Section of General Internal Medicine, 1515 Holcombe Blvd., Box 40, Houston, TX 77030-4095. ences for the adverse effects of aspirin, and on the level of beneficial effects of aspirin on cardiovascular-related mortality.

KEY WORDS: aspirin; primary prevention; cardiovascular disease; decision analysis; risk stratification. J GEN INTERN MED 1998;13:824-835.

C ardiovascular disease is the main cause of morbidity and mortality in the adult Western population, for both men and women. ${ }^{1}$ Strategies for counteracting epidemiologic risk factors such as cigarette smoking, hypertension, and hypercholesterolemia have proved effective as primary and secondary prevention. ${ }^{1}$ Aspirin plays an important role in secondary prevention by reducing the occurrence of cardiovascular events such as myocardial infarction (MI), stroke, and cardiovascular-associated mortality. ${ }^{2}$ Aspirin's role in primary prevention, however, is not as clear.

Although aspirin reduces risk of MI in men aged 40 to 84, its use is associated with important adverse effects, and the balance of benefits and harms is still uncertain. The United States Preventive Task Force does not recommend for or against the use of aspirin for primary prevention ("C" recommendation), as there is insufficient evidence that the benefits outweigh the risks. ${ }^{1}$ The recent Antiplatelet Trialists' Collaboration attributed nonstatistically significant benefits to aspirin for the prevention of 4 cardiovascular events for every 1,000 persons treated over a 5 -year period. ${ }^{2}$ These effects combine a significant onethird reduction of nonfatal MIs and a nonsignificant increase in stroke events. Data are still scarce on the net effects of aspirin in patients having different risk profiles, as even the same amount of relative risk changes would have a greater absolute impact on higher-risk patients. At least four randomized, placebo-controlled trials of aspirin as a prophylaxis are currently under way in more than 65,000 subjects. ${ }^{3}$ The recently published thrombosis prevention trial showed that aspirin significantly reduced nonfatal cardiovascular events in high-risk patients by $32 \%$, although there was no difference in fatal events and total strokes. $^{4}$

While we await further results, decision analysis is an appealing technique for evaluating the risks and benefits of taking aspirin for primary prevention of cardiovascular events in cohorts with different cardiovascular risks. We created a decision-analytic model taking into consideration individual risk profiles for cardiovascular events to determine aspirin's effectiveness in groups of persons with particular sets of risk factors. In this way, we simulated the potential effects of aspirin in a variety of patients seen in the primary care setting. 


\section{METHODS}

\section{Strategies}

We considered two plausible strategies: "aspirin prophylaxis" (aspirin) and "no aspirin prophylaxis" (no aspirin). The first, a primary prevention strategy, consists of lowdose aspirin taken daily (75-325 mg per day). ${ }^{2}$ The second is the "control" strategy.

\section{Markov Decision Model}

We constructed a Markov decision-analytic model ${ }^{5}$ to describe and compare the possible outcomes of the two strategies for patients with any risk profile (see Fig. 1.) The hypothetical cohorts consist of patients with specific cardiovascular risk profiles who all begin in the same state of health (well), and are followed for a defined period of time, during which their health state can change. We chose 1 year for the cycle length of the Markov process for model simplicity but also because it is unlikely for patients to make transitions through several health states in a single year.

In persons with no history of coronary heart disease
(CHD) or cerebrovascular disease, the initial decision is a choice between aspirin treatment and no treatment. Figure 1 shows the square decision node that represents the choice between these options. The chance events related to each choice are represented by Markov subtrees depicted in Figure 1 as the nodes represented by "M." The health states presented in Figure 1 are as follows:

Well aspirin: the state for patients who have no adverse events (MI, bleed, stroke, ulcer, or death) and the initial state for all patients in the aspirin Markov cohort.

Well no aspirin: the health state for subjects with no adverse events and the initial state for all patients in the no aspirin Markov cohort. This is also the state for patients in the aspirin Markov cohort after they have entered the ulcer or bleed state, as aspirin is contraindicated after these situations.

Bleed STM (short-term morbidity): a temporary health state for subjects with nonfatal noncerebral bleeding that requires transfusion. After 1 year in this state, the patient begins the next year in the well no aspirin cohort. (Suspension of aspirin therapy after this adverse effect is normal practice.)

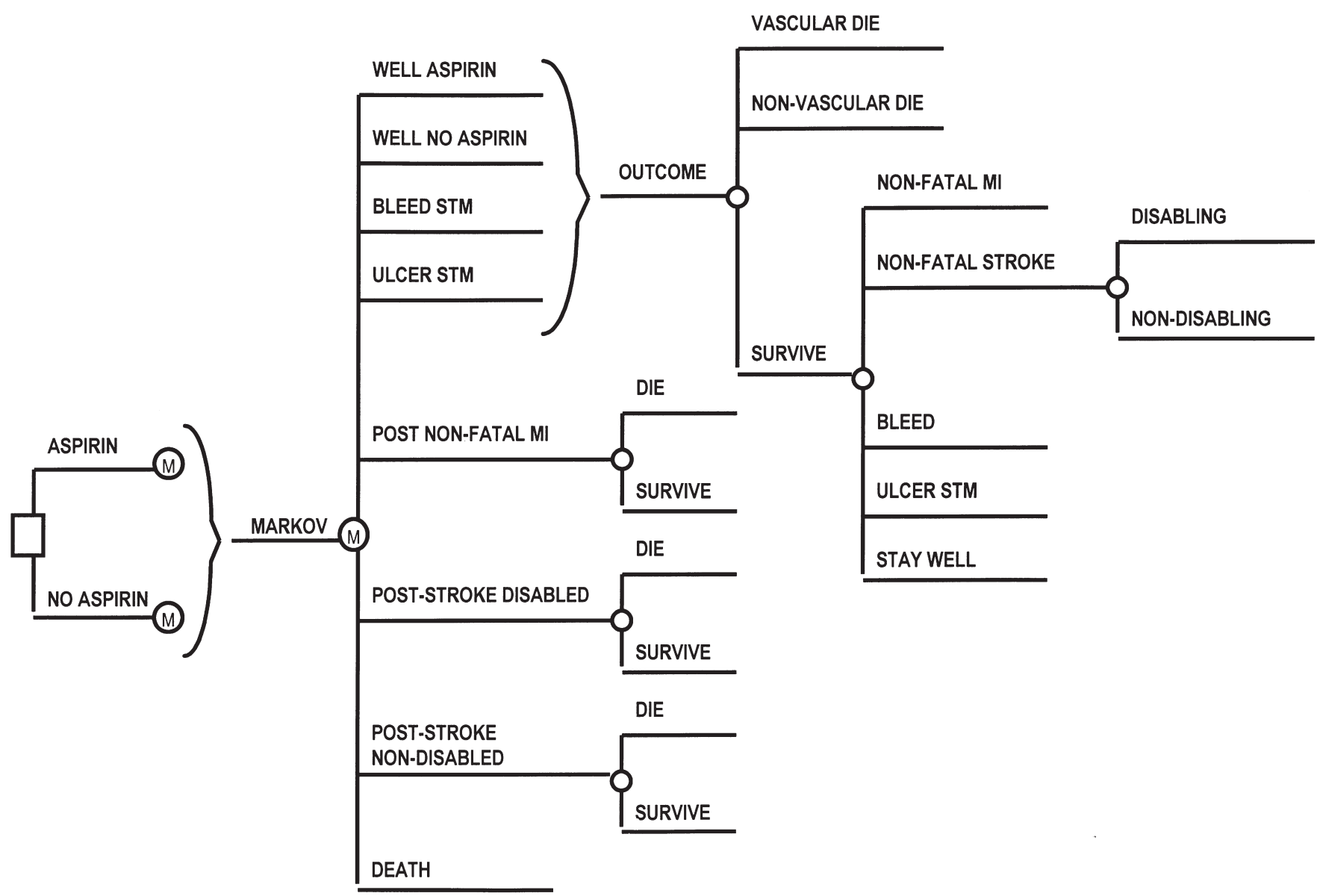

FIGURE 1. Decision tree, including Markov process, for the decision whether or not to take aspirin for primary prevention of cardiovascular events. STM indicates short-term morbidity; MI, myocardial infarction. 
Ulcer STM (short-term morbidity): another temporary health state in which the patient remains for only 1 year and then begins the next year in the well no aspirin cohort, as an episode of active ulcer is also a contraindication to daily aspirin therapy.

Poststroke well: health state of the patient who is alive 1 year after a nondisabling stroke.

Poststroke disabled: health state of the patient who is alive 1 year after a disabling stroke.

Post-Nonfatal myocardial infarction: health state of the patient who survives an MI and remains alive for at least 1 year after the event.

Death: refers to death from cardiovascular or noncardiovascular-related events.

Figure 1 also displays the subtrees representing the chance events for patients during each year of the Markov simulation. Regardless of treatment option aspirin or no aspirin, all cohorts begin the Markov process in one of the well states (well aspirin or well no aspirin, respectively). During each year of the model, the patients may die of age-gender-specific noncardiovascular causes or of riskprofile-specific cardiovascular causes-these patients exit the model by entering the death state. The majority of patients do not have any cardiovascular events; those who do and survive may have had an MI (nonfatal MI), a stroke (nonfatal stroke), a bleed, or an ulcer.

Patients with ulcers and bleeds go through the same tree for the next year, but have a reduced quality of life (see Utilities section below), as they are in a less-thanperfect state of health. If they remain event-free at the end of the year, they go to the well no aspirin state; those previously in the aspirin cohort will no longer receive aspirin.

A person who begins the year in the stroke well, stroke disabled, or nonfatal MI health state can either die of any cause during that year or survive. We also assumed equal probabilities for each branch in both the aspirin and no aspirin Markov trees because we did not want to differentiate primary from secondary prevention effects. It is well proved that aspirin has a clear benefit in secondary prevention, ${ }^{2}$ and all patients who suffer an ischemic stroke or an MI should take aspirin if they have no contraindications.

\section{Cohorts for Analysis}

We considered several cohorts for analysis, including cohorts of typical patients and those with various number and types of risk factors. The cohorts of patients with representative sets of risks profiles are described in Table 1. We chose these cohorts because they represent a set of diverse risk profiles typically encountered by physicians in the asymptomatic population. We modeled seven cohorts for each of four strata based on starting age (55 and 65 years old) and gender (male and female), with each cohort generated by a different numbers of risk factors. The lowestrisk cohort has no risk factors. It incorporates the median population values for cholesterol level, high-density lipoprotein level (HDL), and systolic blood pressure (SBP) according to age and gender and were free from cigarette smoking, diabetes, and left ventricular hypertrophy (LVH). We generated six more cohorts for each age-gender stratum by adding one risk factor in the following order: high cholesterol, low HDL, high SBP (for the previous risk factors we used the 95th percentile for each age-gender strata), cigarette smoking, diabetes, and LVH. The median and 95thpercentile values for risk factors for different age and gender were obtained from Framingham heart study data. ${ }^{6}$ The risk profiles demonstrate a small part of the model's flexibility; certainly many more risk profiles are plausible.

The simulations were run for 10 years; therefore, the maximum attainable outcome is 10 quality-adjusted life years.

\section{Assumptions}

The model incorporates the following assumptions. First, the model applies to persons with no previous history of CHD or cerebrovascular disease, peptic ulcer, or hypersensitivity to aspirin (inclusion criteria of most studies), ${ }^{7,8}$

Table 1. Seven Cohorts Chosen for Each Age (55 and 65 Years Old) and Gender Strata to Represent Various Degrees of Risk*

\begin{tabular}{|c|c|c|c|c|c|c|}
\hline Cohort & $\begin{array}{c}\text { High } \\
\text { Cholesterol } \\
\text { Level }\end{array}$ & $\begin{array}{c}\text { Low HDL } \\
\text { Cholesterol } \\
\text { Level }\end{array}$ & $\begin{array}{c}\text { High } \\
\text { Systolic } \\
\text { Blood } \\
\text { Pressure }\end{array}$ & $\begin{array}{l}\text { Cigarette } \\
\text { Smoking }\end{array}$ & $\begin{array}{l}\text { Diabetes } \\
\text { Mellitus }\end{array}$ & $\begin{array}{c}\text { Left } \\
\text { Ventricular } \\
\text { Hypertrophy }\end{array}$ \\
\hline 1 & - & - & - & - & - & - \\
\hline 2 & + & - & - & - & - & - \\
\hline 3 & + & + & - & - & - & - \\
\hline 4 & + & + & + & - & - & - \\
\hline 5 & + & + & + & + & - & - \\
\hline 6 & + & + & + & + & + & - \\
\hline 7 & + & + & + & + & + & + \\
\hline
\end{tabular}

*The symbol "+" indicates risk factor is present in cohort; "-” indicates risk factor is absent in cohort. 
and only to those individuals aged 55 years or older (a Framingham stroke equation requirement). ${ }^{9}$ Although no randomized controlled trial of primary prevention in women has been conducted, there are enough data from secondary prevention $^{2}$ and prospective cohort studies ${ }^{10}$ to assume similar relative effects in men and women.

The initial follow-up in primary prevention trials was 5 to 6 years. Aspirin effects are similar for each year of follow-up. Because there is no evidence that aspirin stops being effective while it is being taken, ${ }^{2}$ we extended the follow-up period to 10 years. Annual incidences of CHD and cerebrovascular disease were calculated from the Framingham equations, a validated set of equations to predict coronary heart disease and stroke incidence for different risk profiles. ${ }^{1-13}$ It was calculated from each 10-year incidence estimate, assuming a constant transition rate during that period.

Relative effects of aspirin are computed irrespective of patient age, blood pressure, diabetes status, cigarette smoking, HDL and total cholesterol level, and other risk factors. ${ }^{2}$ Aspirin dose was assumed to be 75-325 mg a day, which consequently lowered the rate of bleeding and gastrointestinal complications without compromising aspirin's antiischemic effects. It has not yet been possible to dissociate aspirin's beneficial antithrombotic effects from the deleterious bleeding complications, even with a dose of $75 \mathrm{mg}$ per day. ${ }^{14}$

Strokes were counted in the studies only if they lasted longer than 24 hours and were categorized by severity as disabling or nondisabling based on the effect on function. ${ }^{7}$ Therefore, we restricted our model to strokes other than transient ischemic attacks. Major bleeds are noncerebral bleeds requiring transfusion.

Although aspirin ingestion and colon cancer prevention were linked in some studies, ${ }^{15}$ we did not incorporate this relation into our model because no randomized controlled trials have yet reported such benefits. Minor bleeds were not directly considered in the model; rather, they are incorporated into the preferences for the health state of taking aspirin.

Primary prevention study groups were analyzed with the intention to treat (i.e., we did not explicitly incorporate compliance into the model). However, it should be noted that after 60.2 months, compliance at the end of the Physician's Health Study was $86 \%$ in both the aspirin group ( $n=11,037$, dose of $325 \mathrm{mg}$ on alternate days) and placebo group $(n=11,034) .{ }^{7}$ After 36 months of follow-up in the British study, compliance was $70 \%$ in the aspirin group ( $\mathrm{n}=3,429$, dose of $500 \mathrm{mg}$ per day) and $98 \%$ in the no-aspirin group. ${ }^{8}$

As cardiovascular events present the main cause of death in adults, they have an important impact on total mortality. Among people with more risk factors, total mortality will be higher, mainly because of cardiovascular deaths but sometimes from other causes such as smokingrelated lung cancer. Cardiovascular-related mortality was calculated as risk-factor dependent, but we made the as- sumption that noncardiovascular mortality was age and gender specific without accounting for the differences in risk factors.

\section{Probabilities}

Probability values for the transitions between Markov health states were based on values from the medical literature. Definitions of transition probabilities and their sources are shown in Table 2. Aspirin-related probabilities were extracted mainly from the Antiplatelet Trialists' Collaboration meta-analysis. ${ }^{2}$ Data not available from that study, were provided by the Physician's Health Study, such as the risk of ulcer or disabling stroke. ${ }^{7}$ To estimate the incidence of any event that occurs while taking aspirin, we multiplied the incidence of that same event without taking aspirin by the appropriate relative risk (Table 3 ).

The decision-analytic model was built with the flexibility to accommodate any set of risk profiles. Therefore, we obtained CHD and cerebrovascular disease incidence estimates associated with each risk factor. Framingham equations were used to quantify a cohort's specific CHD risk according to its risk profile. ${ }^{6}$ The risk of CHD is based on the patient's blood pressure, smoking status, total cholesterol and HDL cholesterol levels, presence of electrocardiographic LVH, age, and presence of diabetes. Two Cox parametric regression models were developed, one for men and one for women, and are composed of slightly different parameters reflecting the different relative contributions of these factors for men and women. The equations have also been validated in other populations with a high degree of accuracy. ${ }^{13}$ Excluded from the analysis was angina pectoris, which accounts for about $40 \%$ of first coronary events, ${ }^{16}$ because we were interested in occlusive coronary events and wanted to match our data with those of the Antiplatelet Trialists' Collaboration meta-analysis. ${ }^{2}$ We calculated the 10-year incidence rates of CHD for each particular risk profile and then computed annual incidence rates, assuming a constant rate. ${ }^{17}$

Probabilities of MI and cardiac arrest for the first CHD event by age, gender, annual mortality rates, and annual mortality after surviving the first year were extracted from a CHD computing simulation model developed by Weinstein et al. ${ }^{18}$ and from other epidemiologic studies. ${ }^{19}$

Similar regression models had been developed to estimate the likelihood of stroke for men and women. ${ }^{9}$ Risk factors include age, SBP, presence of hypertension treatment, diabetes, smoking, electrocardiographic LVH, cardiovascular disease, and atrial fibrillation. The model is then used to determine the likelihood of a cohort with a given set of risk factors developing a stroke in a given time period (10 years in our model). As our model was based on primary prevention, all patients started with no cardiovascular disease or atrial fibrillation. Incidence of stroke severity and annual fatality rates were extracted from several epidemiological studies ${ }^{20-24}$ and primary prevention studies. ${ }^{7,8}$ 
Table 2. Probabilities Used in the Decision-Analytic Model*

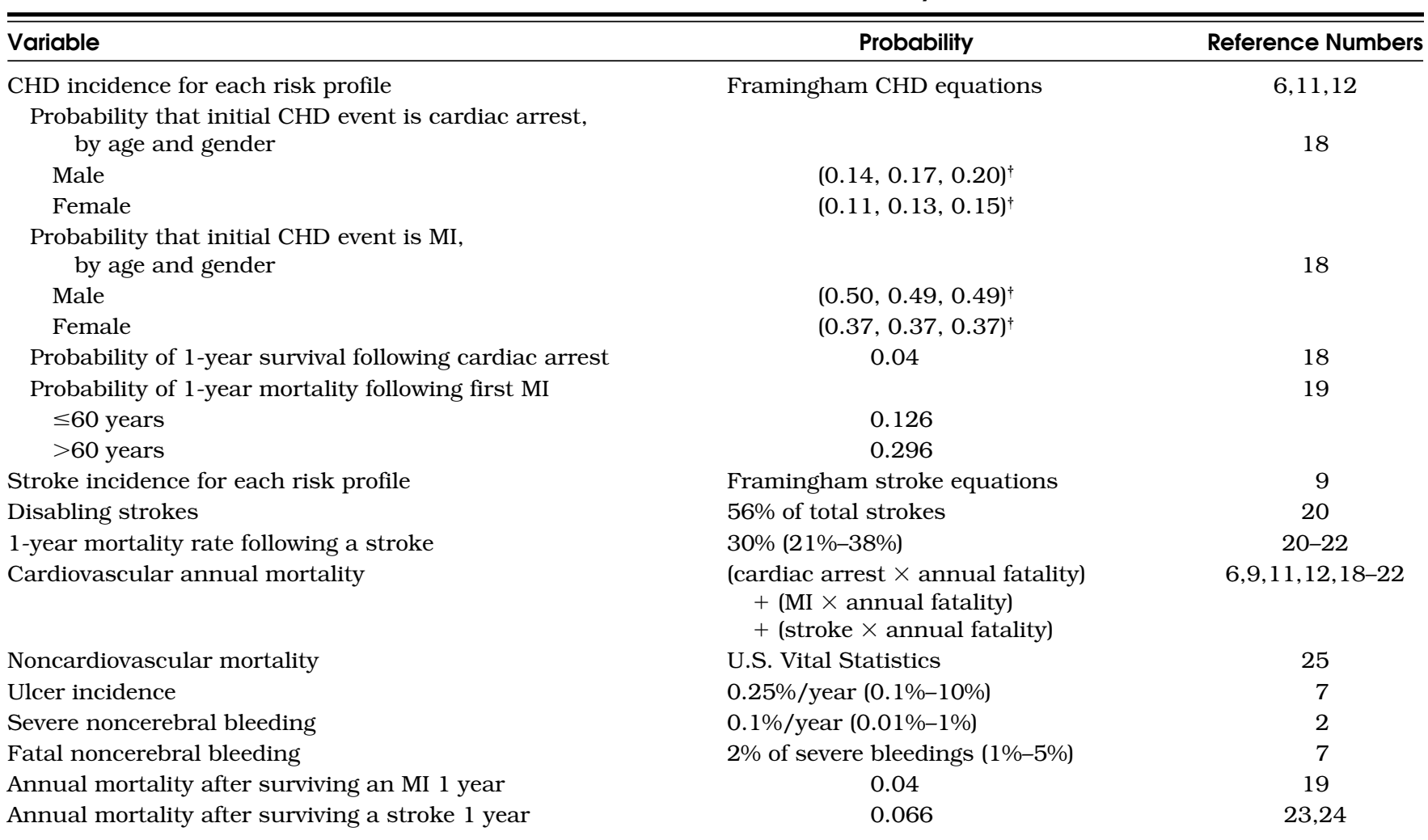

* CHD indicates congestive heart disease; MI, myocardial infarction.

${ }^{\dagger}$ Arrays of three numbers refer to the three age ranges $(55-64,65-74,75-84$ years). These probabilities are constant for every cohort.

We estimated ulcer and major noncerebral bleeding incidence rates from the control arm of the Physicians' Health Study. Caution is needed in interpreting these data as approximately one third of recruited subjects were excluded in the selection process, mainly because of gastrointestinal symptoms. ${ }^{7}$ We accounted for this in the sensitivity analysis.

Age-specific annual mortality rates for the cohorts were taken from U.S. life-table data. ${ }^{25}$

\section{Utilities}

We measured outcomes in quality-adjusted life years. Each Markov health state is assigned a utility value between 0 and 1 . The utility associated with spending 1 year in a given state is the incremental utility. The qualityadjusted survival accrued for the entire Markov process is calculated by multiplying the total number of years spent in each state by the incremental utility of each state and summing the results for each strategy. The Markov model therefore yields a patient prognosis in terms of qualityadjusted survival, up to a possible maximum of 10 qualityadjusted life years.

Table 4 presents a list of utilities and plausible ranges for the various health states. The same utilities were used for every cohort. Taking into consideration the quality of life after a stroke and being consistent with other decision- analytic studies, ${ }^{26}$ we assigned a utility of 0.50 for disabling strokes (hemorrhagic or ischemic) and 0.75 for nondisabling strokes. ${ }^{27}$ For bleeds that result in only short-term morbidity, a utility of 0.97 was assigned for the 1-year period after the event (i.e., 1 week deducted from overall survival). ${ }^{26}$ The utility for the health state of MI was taken from the survey by Tsevat et al. ${ }^{28}$ The utility for the health state of ulcer was taken from a study that evaluated the perceived patient burden of gastrointestinal complications. ${ }^{29}$

Table 3. Effects of Aspirin Assumed in the Decision-Analytic Model

\begin{tabular}{lcc}
\hline Event & $\begin{array}{c}\text { Relative Risk of Different } \\
\text { Events in Patients } \\
\text { Taking Aspirin* }\end{array}$ & $\begin{array}{c}\text { Reference } \\
\text { Number }\end{array}$ \\
\hline $\begin{array}{l}\text { Nonfatal myocardial } \\
\quad \text { infarction }\end{array}$ & $0.77(0.68-0.88)$ & 2 \\
Nonfatal stroke & $1.17(0.94-1.40)$ & 2 \\
Cardiovascular death & $0.98(0.80-1.16)$ & 2 \\
$\quad$ Major nonfatal & & \\
$\quad$ noncerebral bleed & $1.75(1.09-2.69)$ & 7 \\
Ulcer & $1.22(1.0-2.0)$ & 7 \\
\hline
\end{tabular}

* The numbers in parentheses are plausible ranges of the incidence rates. The incidence of events for the no-aspirin cohort was calculated from the Framingham equations ${ }^{6,11-13}$ according the risk profiles and other probabilities shown in Table 2. 
Table 4. Utilities for the Decision-Analytic Model

\begin{tabular}{llc}
\hline \hline Health Status & \multicolumn{1}{c}{ Utility (Range) } & $\begin{array}{c}\text { Reference } \\
\text { Number }\end{array}$ \\
\hline $\begin{array}{l}\text { Nonfatal myocardial } \\
\quad \text { infarction }\end{array}$ & $0.88(0.84-0.93)$ & 28 \\
Disabling stroke & $0.50(0.30-0.70)$ & 26,27 \\
Nondisabling stroke & $0.75(0.7-1.00)$ & 27 \\
Ulcer & $0.91(0.865-0.96)$ & 29 \\
Noncerebral bleed & $0.97(0.8-1.00)$ & 26 \\
Taking aspirin & $0.999(0.985-1.0)$ & 26
\end{tabular}

The side effects of taking medication can diminish the quality of life. The utility of taking one aspirin a day is assumed to be 0.999 . This value is consistent with assumptions made in previous decision analyses comparing management strategies for atrial fibrillation. ${ }^{26,27}$

\section{Calculations}

The model was developed using SMLTREE 2.9 software for decision analysis (J.P. Hollenberg, New York, N.Y., 1989). One-way sensitivity analysis was performed to examine the robustness of the optimal decision to variations in both probability and utility estimates.

We performed a sensitivity analysis on the uncertain probabilities in the model, such as the annual probability of having an ulcer or a major bleed. We also performed a threshold analysis on the utility of taking aspirin, as this burden may vary from patient to patient. Finally, we examined the thresholds associated with aspirin's relative effects on the different outcomes.

\section{RESULTS}

The majority of cohorts achieved some benefit from aspirin. However, in all the cohorts, regardless of age and gender, we found that the decision to use aspirin as a chemoprophylactic agent depended on the risk profile (Tables 5 and 6). The model suggested that aspirin not be taken in the cohorts with the lowest-risk profile (loss of 1.83 quality-adjusted days in 10 years of follow-up) and favored taking aspirin in the cohorts with higher-risk profiles (gain of 3.3 to 11.3 quality-adjusted life days in 10 years of follow-up). Regarding aspirin's effect in crude life expectancy, all cohorts benefited from receiving aspirin with a net benefit ranging from 0.73 to 8.04 days (see Tables 5 and 6). In general, the benefit of aspirin was greater as the number of risk factors increased. The presence of LVH had different effects among the different cohorts; it increased the benefit of aspirin in 55-year-old women but diminished the benefit of aspirin in all other cohorts. This seemingly contradictory effect could be explained by LVH's significant effect on stroke incidence and relatively low incidence of stroke in 55-year-old women.

We tested the stability of the model performing oneway sensitivity analysis on the probabilities that are less certain in the literature (annual ulcer incidence and major bleeds) (Table 7). The model was robust for most of the ranges of aspirin's relative effects on probabilities (nonfatal MI, ulcer, and major bleeding). The model was also robust for the ranges of most utilities (nonfatal MI, disabling and nondisabling stroke, ulcer, and major bleeding). In the cohorts of 65-year-old men and women (data not shown for women), the optimal strategy to take aspirin was also sensitive to aspirin's effect on stroke incidence.

Table 5. Difference in 10-Year Survival in 55-Year-Old Cohorts*

\begin{tabular}{|c|c|c|c|c|c|c|}
\hline Cohort & $\begin{array}{c}\text { Life } \\
\text { Expectancy } \\
\text { for Aspirin }\end{array}$ & $\begin{array}{c}\text { Life } \\
\text { Expectancy } \\
\text { for No Aspirin }\end{array}$ & $\begin{array}{l}\text { Difference } \\
\text { in Life } \\
\text { Expectancy, } \\
\text { Days }\end{array}$ & $\begin{array}{c}\text { QALE } \\
\text { for } \\
\text { Aspirin }\end{array}$ & $\begin{array}{c}\text { QALE } \\
\text { for } \\
\text { No Aspirin }\end{array}$ & $\begin{array}{c}\text { Difference } \\
\text { in QALE, } \\
\text { Quality-Adjusted } \\
\text { Days }\end{array}$ \\
\hline \multicolumn{7}{|l|}{ Men } \\
\hline 1. No risk factors & 9.487 & 9.481 & 2.19 & 9.437 & 9.438 & -0.37 \\
\hline 2. $\uparrow$ Chol & 9.427 & 9.419 & 2.92 & 9.369 & 9.364 & 1.83 \\
\hline 5. $\uparrow$ Chol, $\downarrow$ HDL, $\uparrow$ SBP, CIGAR & 9.022 & 9.003 & 6.94 & 8.875 & 8.847 & 10.23 \\
\hline 6. $\uparrow$ Chol, $\downarrow$ HDL, $\uparrow$ SBP, CIGAR, DM & 8.902 & 8.882 & 7.30 & 8.723 & 8.694 & 10.59 \\
\hline 7. $\uparrow$ Chol, $\downarrow_{\mathrm{HDL}}, \uparrow \mathrm{SBP}, \mathrm{CIGAR}, \mathrm{DM}, \mathrm{LVH}$ & 8.476 & 8.457 & 6.94 & 8.173 & 8.145 & 10.23 \\
\hline \multicolumn{7}{|l|}{ Women } \\
\hline 1. No risk factors & 9.705 & 9.703 & 0.73 & 9.680 & 9.685 & -1.83 \\
\hline 6. $\uparrow$ Chol, $\downarrow_{\mathrm{HDL}} \uparrow \mathrm{SBP}, \mathrm{CIGAR}, \mathrm{DM}$ & 9.275 & 9.258 & 6.21 & 9.164 & 9.142 & 8.04 \\
\hline 7. $\uparrow$ Chol, $\downarrow_{\mathrm{HDL}}, \uparrow \mathrm{SBP}, \mathrm{CIGAR}, \mathrm{DM}, \mathrm{LVH}$ & 8.949 & 8.927 & 8.04 & 8.758 & 8.727 & 11.32 \\
\hline
\end{tabular}

*The optimal strategy is depicted in boldface type. QALE indicates quality-adjusted life expectancy; Chol, cholesterol level; HDL, high density lipoprotein cholesterol level; SBP, systolic blood pressure; CIGAR, cigarette smoking; DM, diabetes mellitus; LVH, left ventricular hypertrophy. 
Table 6. Difference in 10-Year Survival in 65-Year-Old Cohorts*

\begin{tabular}{|c|c|c|c|c|c|c|}
\hline Cohort & $\begin{array}{c}\text { Life } \\
\text { Expectancy } \\
\text { for Aspirin }\end{array}$ & $\begin{array}{c}\text { Life } \\
\text { Expectancy } \\
\text { for No Aspirin }\end{array}$ & $\begin{array}{c}\text { Difference } \\
\text { in Life } \\
\text { Expectancy, } \\
\text { Days } \\
\end{array}$ & $\begin{array}{c}\text { QALE } \\
\text { for } \\
\text { Aspirin }\end{array}$ & $\begin{array}{c}\text { QALE } \\
\text { for } \\
\text { No Aspirin } \\
\end{array}$ & $\begin{array}{c}\text { Difference } \\
\text { in QALE, } \\
\text { Quality-Adjusted } \\
\text { Days }\end{array}$ \\
\hline \multicolumn{7}{|l|}{ Men } \\
\hline No risk factors & 8.907 & 8.896 & 4.02 & 8.837 & 8.835 & 0.73 \\
\hline$\uparrow$ Chol & 8.802 & 8.792 & 3.65 & 8.723 & 8.716 & 2.56 \\
\hline$\uparrow$ Chol, $\downarrow$ HDL, $\uparrow$ SBP, CIGAR & 8.208 & 8.193 & 5.48 & 8.029 & 8.012 & 6.21 \\
\hline$\uparrow$ Chol, $\downarrow_{\mathrm{HDL}}, \uparrow \mathrm{SBP}, \mathrm{CIGAR}, \mathrm{DM}$ & 8.041 & 8.027 & 5.11 & 7.821 & 7.806 & 5.48 \\
\hline 个Chol, $\downarrow$ HDL, $\uparrow$ SBP, CIGAR, DM, LVH & 7.488 & 7.480 & 2.92 & 7.112 & 7.114 & -0.73 \\
\hline \multicolumn{7}{|l|}{ Women } \\
\hline No risk factors & 9.378 & 9.375 & 1.10 & 9.343 & 9.348 & -1.83 \\
\hline$\uparrow \mathrm{Chol}, \downarrow$ HDL, $\uparrow \mathrm{SBP}, \mathrm{CIGAR}, \mathrm{DM}$ & 8.725 & 8.710 & 5.48 & $\mathbf{8 . 5 8 2}$ & 8.569 & 4.75 \\
\hline$\uparrow$ Chol, $\downarrow$ HDL, $\uparrow$ SBP, CIGAR, DM, LVH & 8.251 & 8.235 & 5.84 & 7.993 & 7.984 & 3.29 \\
\hline
\end{tabular}

*The optimal strategy is depicted in boldface type. QALE indicates quality-adjusted life expectancy; Chol, cholesterol level; HDL, high density lipoprotein cholesterol level; SBP, systolic blood pressure; CIGAR, cigarette smoking; DM, diabetes mellitus; LVH, left ventricular hypertrophy.

Table 7. Threshold Values for Comparison Between Treatment Strategies for Cohorts of 65-Year-Old Men and 55-Year-Old Women*

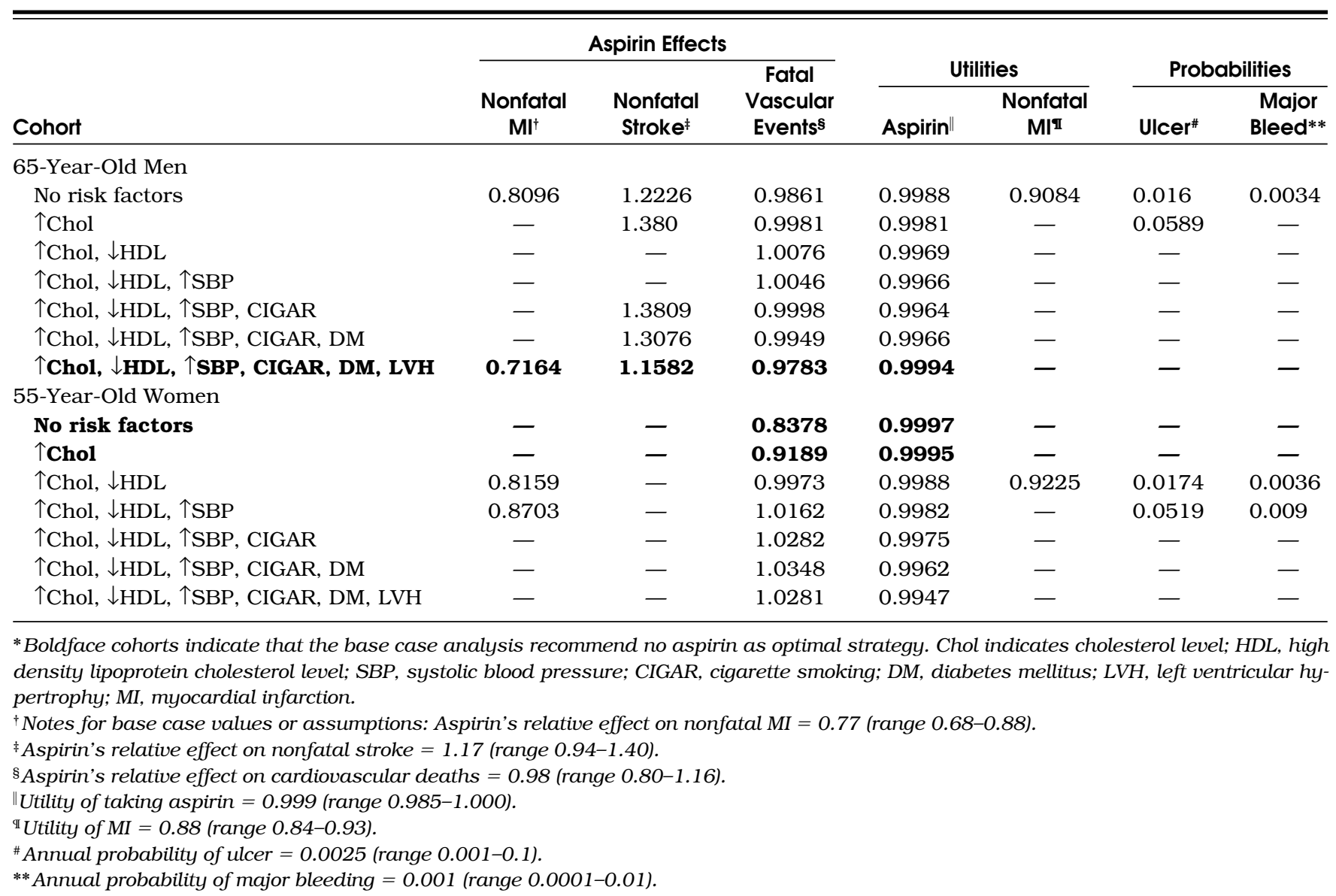


Two variables were extremely significant in one-way sensitivity analysis: the utility of taking aspirin and aspirin's relative effect on cardiovascular-related mortality. All the cohorts tested were sensitive to the utility of taking aspirin and to aspirin's effect on cardiovascular mortality. To show the pattern of variables that were sensitive in the different cohorts, Table 7 demonstrates a threshold analysis of key variables in 65-year-old male cohorts and in 55-year-old female cohorts.

The interpretation of the results in Table 7 is dependent on the optimal decision recommended by the model, the type of variable under examination by the one-way sensitivity analysis (i.e., probability of event, relative risk of aspirin's effects, or utility of adverse outcome), and whether or not a threshold exists.

For the rows not in boldface type, the baseline analysis recommended taking aspirin. In these scenarios, if a threshold value for the probability of an event or the relative risk of aspirin's effects does not exist, then the optimal decision to take aspirin is robust to variation in the parameter under examination. Alternatively, if a threshold value for the probability of an event or the relative risk of aspirin's effects does exist, then for any value greater than the threshold, not taking aspirin would become the optimal decision.

\section{Aspirin's Effect on Relative Risk}

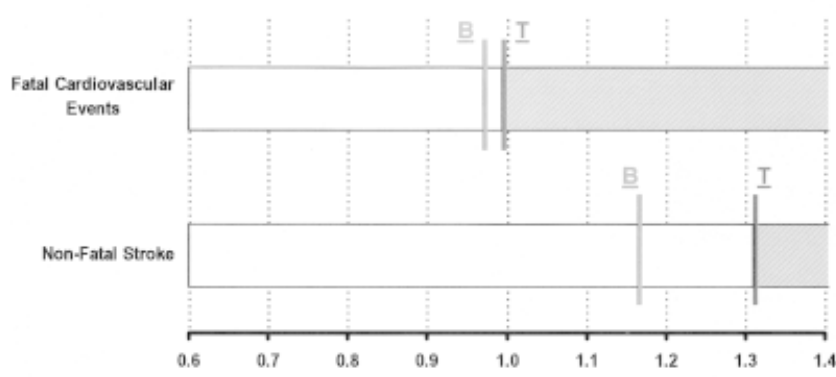

$\underline{\text { Utilities }}$

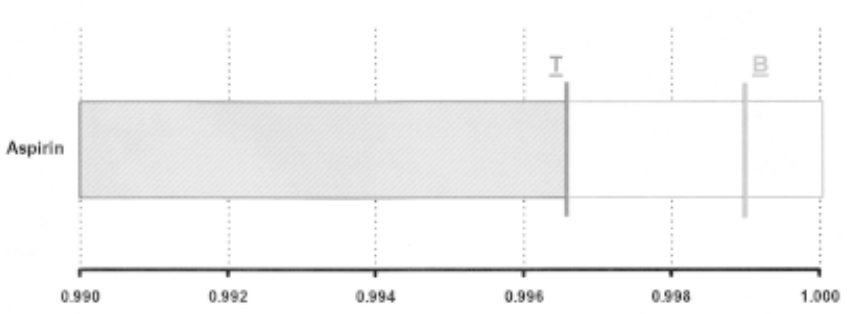

$$
\begin{aligned}
& \text { Aspirin is optimal strategy } \\
& \text { No Aspirin is optimal strategy }
\end{aligned}
$$

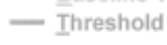

FIGURE 2. Threshold analysis: 65-year-old men with five risk factors. Shaded region indicates that no aspirin is optimal strategy.
Similar reasoning can be made for utility thresholds when the baseline analysis recommended taking aspirin. However, for utilities there are two different effects-one for the burden (disutility) of taking aspirin, and the other for the burden of MI. If a threshold exists for the utility of taking aspirin, then a decrease in the utility of that health state to the threshold or lower will change the optimal decision to not take aspirin. If the utility for non-fatal MI becomes greater than the threshold for that variable, then again the recommendation changes to not take aspirin.

For example, in the cohort of the next-to-highest-risk 65-year-old men (sixth row of top part of Table 7), the baseline analysis indicated that aspirin was the optimal strategy. Figure 2 demonstrates the threshold analysis. For utility values smaller than 0.9966, rather than the base case value of 0.999 , the no-aspirin strategy would be recommended. In that same cohort, if the relative risk of stroke while taking aspirin were greater than 1.3075, then the noaspirin strategy would be recommended (compare with base case, for which relative risk of stroke equals 1.17).

The logic is reversed when a cohort's optimal strategy in the baseline analysis was to not take aspirin, designated by the boldface rows in Table 7. Again, the interpretation of the threshold is dependent on the variable that is examined in the one-way sensitivity analysis. For the threshold for the

\section{Aspirin's Effect on Relative Risk}

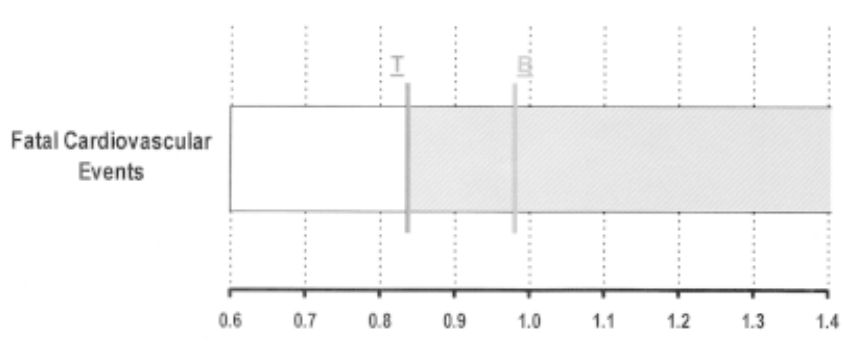

\section{$\underline{\text { Utilities }}$}
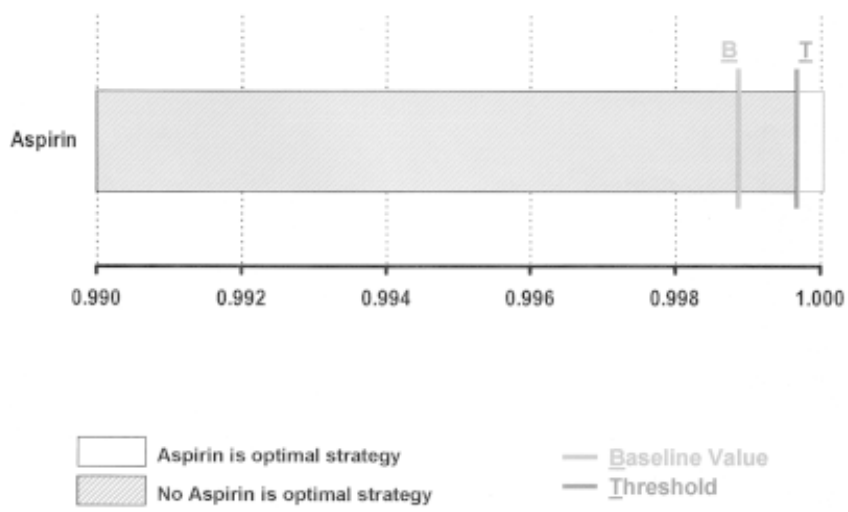

FIGURE 3. Threshold analysis: 55-year-old women with no risk factors. Shaded region indicates that no aspirin is optimal strategy. 
Table 8. Aspirin Primary Prevention Table of Risk Factors for Men*

\begin{tabular}{|c|c|c|c|c|c|c|c|c|c|c|c|c|c|c|c|}
\hline \multicolumn{4}{|c|}{ Smoke } & \multicolumn{4}{|c|}{ No Smoke } & \multicolumn{4}{|c|}{ Smoke } & \multicolumn{4}{|c|}{ No Smoke } \\
\hline LVH+ & LVH- & LVH+ & LVH- & LVH+ & LVH- & LVH+ & LVH- & LVH+ & LVH- & LVH+ & LVH- & LVH+ & LVH- & LVH+ & LVH- \\
\hline $\mathbf{A}$ & $\mathbf{A}$ & $\mathbf{A}$ & $\mathbf{A}$ & $\mathbf{A}$ & $\mathbf{A}$ & $\mathbf{A}$ & $\mathbf{A}$ & $\mathbf{A}$ & A & $\mathbf{A}$ & A & $\mathbf{A}$ & A & $\mathbf{A}$ & A \\
\hline $\mathrm{N}$ & A & $\mathrm{N}$ & $\mathbf{A}$ & A & $\mathbf{A}$ & $\mathbf{A}$ & $\mathbf{A}$ & $\mathrm{N}$ & $\mathrm{N}$ & $\mathrm{N}$ & A & $\mathrm{N}$ & A & A & A \\
\hline
\end{tabular}

*Boldface indicates aspirin strategy has benefit greater than 3.5 quality-adjusted life days; A, benefit of aspirin strategy is smaller than 3.5 quality-adjusted life days; $N$, no aspirin is better than aspirin (difference always smaller than 1.8 quality-adjusted life days); DM, diabetes mellitus; LVH, left ventricular hypertrophy. ${ }_{\dagger}$ Hypertension is systolic blood pressure of 170 .

${ }^{\ddagger}$ Hypercholesterolemia: 95th percentile Framingham (295 mg/dL).

s Normal cholesterol level is $200 \mathrm{mg} / \mathrm{dL}$.

utility of the health state of taking aspirin, the optimal decision would change from not taking aspirin to taking aspirin for any value higher than the threshold. No other probability variable has a threshold for the cohorts originally better off with the no-aspirin strategy. Thus, no reasonable change in these probabilities would change the optimal recommendations for these cohorts. With regard to the cohorts for which the initial choice was no aspirin: if a threshold exists for the effects of aspirin, relative risk variables of effect, a value smaller than the threshold would change the optimal choice from the no-aspirin strategy to the aspirin strategy.

The above reasoning is demonstrated in the threshold analysis for the cohort of lowest-risk 55-year-old women (Fig. 3). In this cohort the baseline analysis showed that no aspirin was the optimal strategy. If the utility of the health state of taking aspirin were 0.9997 or higher, then taking aspirin would be recommended. Similarly, the aspirin strategy would be favored if aspirin's relative risk on fatal vascular events were less than 0.8378 (compare with baseline value for aspirin's relative effect on cardiovascular mortality equal to 0.98).

For the purposes of clinical applicability, we show in Tables 8 and 9 all the possible cohorts and aspirin's potential benefit in each of them. Although in the great majority of possible cohorts the aspirin strategy was better than the no-aspirin strategy, these tables demonstrate the cohorts that most benefit from taking aspirin, as indicated by a boldface $\mathbf{A}$. In these cohorts, the benefit of taking aspirin was equal to or greater than 3.5 quality-adjusted life days, which we considered a clinically relevant difference, especially considering the significant impact that the administration of aspirin could have at the population level.

\section{DISCUSSION}

The role of aspirin in the primary prevention of cardiovascular events, major causes of morbidity and mortality, can have important public health implications. However, this role is controversial and has engendered considerable disagreement among various organizations. According to the United States Preventive Services Task Force, "There is insufficient evidence to determine whether the proven benefits of routine aspirin prophylaxis given for the primary prevention of MI in asymptomatic men aged 40 to 84 years outweigh the proven harms. In men with other risk factors for coronary heart disease who lack contraindications to aspirin use, the benefits may outweigh the harms. In asymptomatic men without risk factors for coronary

Table 9. Aspirin Primary Prevention Table of Risk Factors for Women*

\begin{tabular}{|c|c|c|c|c|c|c|c|c|c|c|c|c|c|c|c|}
\hline \multicolumn{16}{|c|}{ Hypertension ${ }^{\dagger}$} \\
\hline \multicolumn{8}{|c|}{ Hypercholesterolemia ${ }^{\ddagger}$} & \multicolumn{8}{|c|}{ Normal Cholesterol Levels\$ } \\
\hline \multicolumn{4}{|c|}{ Smoke } & \multicolumn{4}{|c|}{ No Smoke } & \multicolumn{4}{|c|}{ Smoke } & \multicolumn{4}{|c|}{ No Smoke } \\
\hline \multicolumn{2}{|c|}{ DM+ } & \multicolumn{2}{|c|}{ DM- } & \multicolumn{2}{|c|}{ DM+ } & \multicolumn{2}{|c|}{ DM- } & \multicolumn{2}{|c|}{ DM+ } & \multicolumn{2}{|c|}{ DM- } & \multicolumn{2}{|c|}{$\mathrm{DM}+$} & \multicolumn{2}{|c|}{ DM- } \\
\hline LVH+ & LVH- & $\mathrm{LVH}+$ & LVH- & $\mathrm{LVH}+$ & LVH- & $\mathrm{LVH}+$ & LVH- & LVH+ & LVH- & $\mathrm{LVH}+$ & LVH- & $\mathrm{LVH}+$ & LVH- & $\mathrm{LVH}+$ & LVH- \\
\hline $\mathbf{A}$ & $\mathbf{A}$ & $\mathbf{A}$ & $\mathbf{A}$ & $\mathbf{A}$ & $\mathbf{A}$ & $\mathbf{A}$ & $\mathbf{A}$ & $\mathbf{A}$ & $\mathbf{A}$ & $\mathbf{A}$ & $\mathbf{A}$ & $\mathbf{A}$ & $\mathbf{A}$ & $\mathbf{A}$ & $\mathbf{N}$ \\
\hline $\mathbf{A}$ & $\mathbf{A}$ & $\mathbf{A}$ & $\mathbf{A}$ & $\mathbf{A}$ & $\mathbf{A}$ & $\mathbf{A}$ & A & $\mathbf{A}$ & A & A & A & $\mathbf{A}$ & A & A & $\mathrm{N}$ \\
\hline $\mathbf{A}$ & $\mathbf{A}$ & $\mathbf{A}$ & $\mathbf{A}$ & $\mathbf{A}$ & $\mathbf{A}$ & $\mathbf{A}$ & A & A & A & A & $\mathrm{N}$ & A & $\mathrm{N}$ & A & $\mathrm{N}$ \\
\hline A & A & A & A & $\mathbf{A}$ & $\mathbf{A}$ & $\mathbf{A}$ & A & $\mathrm{N}$ & $\mathrm{N}$ & $\mathrm{N}$ & $\mathrm{N}$ & $\mathrm{N}$ & $\mathrm{N}$ & $\mathrm{N}$ & $\mathrm{N}$ \\
\hline
\end{tabular}

*Boldface indicates aspirin strategy has benefit greater than 3.5 quality-adjusted life days; A, benefit of aspirin strategy is smaller than 3.5 quality-adjusted life days; $N$, no aspirin is better than aspirin (difference always smaller than 1.8 quality-adjusted life days); DM, diabetes mellitus; LVH, left ventricular hypertrophy. ${ }^{\dagger}$ Hypertension is systolic blood pressure of 170 .

${ }^{\ddagger}$ Hypercholesterolemia is 95th percentile Framingham $(295 \mathrm{mg} / \mathrm{dL}$ ).

sNormal cholesterol level is $200 \mathrm{mg} / \mathrm{dL}$. 
Table 8. Continued

\begin{tabular}{|c|c|c|c|c|c|c|c|c|c|c|c|c|c|c|c|c|}
\hline \multicolumn{16}{|c|}{ No Hypertension ${ }^{\dagger}$} & \multirow{5}{*}{$\begin{array}{l}\text { Age, } \\
\text { Years }\end{array}$} \\
\hline \multicolumn{8}{|c|}{ Hypercholesterolemia ${ }^{\ddagger}$} & \multicolumn{8}{|c|}{ Normal Cholesterol Levels§ } & \\
\hline \multicolumn{4}{|c|}{ Smoke } & \multicolumn{4}{|c|}{ No Smoke } & \multicolumn{4}{|c|}{ Smoke } & \multicolumn{4}{|c|}{ No Smoke } & \\
\hline \multicolumn{2}{|c|}{$\mathrm{DM}+$} & \multicolumn{2}{|c|}{ DM- } & \multicolumn{2}{|c|}{ DM+ } & \multicolumn{2}{|c|}{ DM- } & \multicolumn{2}{|c|}{$\mathrm{DM}+$} & \multicolumn{2}{|c|}{ DM- } & \multicolumn{2}{|c|}{$\mathrm{DM}+$} & \multicolumn{2}{|c|}{ DM- } & \\
\hline LVH+ & LVH- & LVH+ & LVH- & $\mathrm{LVH}+$ & LVH- & LVH+ & LVH- & $\mathrm{LVH}+$ & LVH- & LVH+ & LVH- & LVH+ & $\overline{\text { LVH- }}$ & LVH+ & $\overline{\text { LVH- }}$ & \\
\hline $\mathbf{A}$ & $\mathbf{A}$ & $\mathbf{A}$ & $\mathbf{A}$ & $\mathbf{A}$ & $\mathbf{A}$ & $\mathbf{A}$ & $\mathbf{A}$ & $\mathbf{A}$ & A & $\mathbf{A}$ & A & $\mathbf{A}$ & A & A & $\mathrm{N}$ & 55 \\
\hline $\mathbf{A}$ & $\mathbf{A}$ & $\mathbf{A}$ & $\mathbf{A}$ & $\mathbf{A}$ & $\mathbf{A}$ & $\mathbf{A}$ & $\mathbf{A}$ & $\mathbf{A}$ & A & $\mathbf{A}$ & $\mathrm{A}$ & $\mathbf{A}$ & A & $\mathbf{A}$ & $\mathrm{N}$ & 60 \\
\hline $\mathbf{A}$ & $\mathbf{A}$ & $\mathbf{A}$ & $\mathbf{A}$ & $\mathbf{A}$ & $\mathbf{A}$ & $\mathbf{A}$ & $\mathbf{A}$ & A & A & A & A & A & A & A & $\mathrm{N}$ & 65 \\
\hline A & $\mathbf{A}$ & $\mathbf{A}$ & $\mathbf{A}$ & $\mathbf{A}$ & $\mathbf{A}$ & $\mathbf{A}$ & $\mathbf{A}$ & A & A & A & A & A & $\mathrm{A}$ & A & $\mathrm{N}$ & 70 \\
\hline
\end{tabular}

heart disease or with relative contraindications to aspirin use, the harms may outweigh the benefits." ${ }^{1}$

The Canadian Task Force on Periodic Health Examination states, "The evidence is not strong enough to support a recommendation that routine aspirin therapy be used or not be used for the primary prevention of cardiovascular disease in asymptomatic men. The decision on whether to prescribe aspirin should be made on an individual basis after the benefits of decreased incidence of ischemic cardiovascular events have been balanced against the potential risks associated with prolonged aspirin use."30

The American Heart Association advises, "Any decision to use aspirin in the primary prevention of cardiovascular disease should be based on an individual clinical judgment by a physician or other health care provider in which the cardiovascular risk profile of the patient is considered." ${ }^{31}$ Hirsh et al., however, state that "Aspirin is indicated in asymptomatic men and women over age 50 to prevent myocardial infarction." 32

The latest Antiplatelet Trialists' Collaboration describes the data in this way: Among approximately 28,000 primary prevention subjects studied, allocation to receive an average of just over 5 years of antiplatelet therapy pro- duced a small but highly significant reduction of 5 per 1,000 nonfatal MIs, but the reduction in cardiovascular events (non-fatal MI, nonfatal stroke, and cardiovascular death) was slightly smaller and less clearly significant-4 per 1,000 . In contrast with the highly significant reduction in nonfatal strokes among high-risk subjects, there was no evidence of any decrease in nonfatal strokes among these low-risk subjects; rather, the reverse was found, with 2 per 1,000 increase in nonfatal strokes (though this was not statistically significant). ${ }^{2}$

The above sets of recommendations leave health care providers with a considerable degree of uncertainty. How do we define significant risk for MI? How can we balance the benefits against the risks in a systematic way? What are the specific individual risk profiles that have a net benefit from aspirin therapy? What is the role of aspirin in women? What happens when quality-of-life measures are considered? Answers to these questions are currently being sought in randomized controlled trials, ${ }^{3}$ but are still not available. To bring some systematic appraisal to this conundrum, we constructed a Markov decision model that uses as input the variables of gender, age, total cholesterol level, HDL cholesterol level, SBP, antihypertensive

Table 9. Continued

\begin{tabular}{|c|c|c|c|c|c|c|c|c|c|c|c|c|c|c|c|c|}
\hline \multicolumn{16}{|c|}{ No Hypertension ${ }^{\dagger}$} & \multirow{5}{*}{$\begin{array}{l}\text { Age, } \\
\text { Years }\end{array}$} \\
\hline \multicolumn{8}{|c|}{ Hypercholesterolemia ${ }^{\ddagger}$} & \multicolumn{8}{|c|}{ Normal Cholesterol Levels\$ } & \\
\hline \multicolumn{4}{|c|}{ Smoke } & \multicolumn{4}{|c|}{ No Smoke } & \multicolumn{4}{|c|}{ Smoke } & \multicolumn{4}{|c|}{ No Smoke } & \\
\hline \multicolumn{2}{|c|}{$\mathrm{DM}+$} & \multicolumn{2}{|c|}{ DM- } & \multicolumn{2}{|c|}{$\mathrm{DM}+$} & \multicolumn{2}{|c|}{ DM- } & \multicolumn{2}{|c|}{$\mathrm{DM}+$} & \multicolumn{2}{|c|}{ DM- } & \multicolumn{2}{|c|}{ DM+ } & \multicolumn{2}{|c|}{ DM- } & \\
\hline LVH+ & LVH- & LVH+ & LVH- & LVH+ & LVH- & LVH+ & LVH- & LVH+ & LVH- & LVH+ & LVH- & LVH+ & LVH- & LVH & LVH- & \\
\hline $\mathbf{A}$ & $\mathbf{A}$ & $\mathbf{A}$ & $\mathbf{A}$ & $\mathbf{A}$ & $\mathbf{A}$ & $\mathbf{A}$ & $\mathbf{A}$ & $\mathbf{A}$ & $\mathbf{A}$ & $\mathbf{A}$ & $\mathbf{N}$ & $\mathbf{A}$ & $\mathbf{N}$ & $\mathbf{A}$ & $\mathbf{N}$ & 55 \\
\hline $\mathbf{A}$ & $\mathbf{A}$ & $\mathbf{A}$ & A & $\mathbf{A}$ & $\mathbf{A}$ & $\mathbf{A}$ & A & $\mathbf{A}$ & A & A & $\mathrm{N}$ & $\mathbf{A}$ & $\mathrm{N}$ & A & $\mathrm{N}$ & 60 \\
\hline $\mathbf{A}$ & $\mathbf{A}$ & $\mathbf{A}$ & A & $\mathbf{A}$ & $\mathbf{A}$ & $\mathbf{A}$ & A & A & A & A & $\mathrm{N}$ & A & $\mathrm{N}$ & A & $\mathrm{N}$ & 65 \\
\hline $\mathbf{A}$ & $\mathbf{A}$ & $\mathbf{A}$ & A & $\mathbf{A}$ & A & $\mathbf{A}$ & A & A & A & A & $\mathrm{N}$ & A & $\mathrm{N}$ & A & $\mathrm{N}$ & 70 \\
\hline
\end{tabular}


treatment, smoking status, diabetes mellitus, and electrocardiographic LVH. This model has the flexibility to accommodate any particular set of risk factors. For this purpose we combined epidemiologic data with aspirin effectiveness data gathered from the Antiplatelet Trialists' Collaboration meta-analysis and utility data from the literature to predict the incidence of cardiovascular events given particular risk factors. It is therefore possible to use this model for physician-patient decision making at the time of an office visit, recognizing that this analysis should be applied only to men and women between the ages of 55 and 70 and that it only follows them for a 10-year period.

The two most important variables to which all the cohorts were sensitive were the utility of aspirin and the effect of aspirin on the risk of fatal vascular events (something that we hope further studies will clarify). For older cohorts, who have a higher stroke incidence, aspirin's relative effect on stroke incidence is also a key variable. Reassuring results regarding stroke incidence were recently published. ${ }^{4}$

There is no clear evidence that the relative effects of aspirin change with risk factor status. Conversely, these relative effects could have a different absolute effect depending on the predicted incidence of cardiovascular events for persons with a particular risk profile.

Taking all this into consideration, aspirin administration for primary prevention in high-risk cohorts compares favorably with other prevention strategies. By comparison, a widely accepted intervention such as mammography screening for women aged 50 to 69 years improves life expectancy 12 days; mammography screening in women aged 40 to 49 years - a much more controversial issue-only adds 2.5 days of life expectancy. ${ }^{33}$ Benefits of aspirin for primary prevention might even be greater if aspirin's beneficial effect in patients with colorectal cancer is included. Our model showed that patient preferences make a difference in the optimal decision. ${ }^{34}$

Our study is more supportive of giving aspirin for primary prevention than the general statement of the United States Preventive Services Task Force. ${ }^{1}$ The model predicts benefits in life expectancy and quality-adjusted life expectancy for most risk profiles, although absolute benefit increases in high-risk patients. The analysis gives the decision maker a quantitative method to decide if a person with a particular risk profile would benefit from aspirin. Although the benefits from aspirin are not large for an individual, they are comparable to those of currently accepted interventions; from a public health perspective, the benefits could be important for men and women between ages 55 and 70. The estimated benefits have been outlined in tabular format for purposes of clinical decision making in the office setting. These tables can assist general internists and their patients in deciding whether aspirin is appropriate based on the patient's age, gender, and risk factors.

This manuscript greatly benefited from clinical and methodologic insights from James Dolan, MD. Diane Rivera provided editorial assistance, and Monica Hamlin provided secretarial support for this manuscript. In addition, the authors would like to thank both The University of Texas Medical Branch at Galveston and Unidad de Medicina Familiar del Hospital Italiano de Buenos Aires for their support of Dr. Augustovski during the time which this project was conducted.

\section{REFERENCES}

1. United States Preventive Task Force. Guide to Clinical Preventive Services. 2nd ed. Baltimore, Md: Williams and Wilkins; 1996.

2. Antiplatelet Trialists' Collaboration. Collaborative overview of randomized trials of antiplatelet therapy, I: prevention of death, myocardial infarction, and stroke by prolonged antiplatelet therapy in various categories of patients. BMJ. 1994;308:81-106.

3. Patrono C. Aspirin as an antiplatelet drug. N Engl J Med. 1994; 330:1287-94.

4. The Medical Research Council's General Practice Research Framework. Thrombosis prevention trial: randomised trial of low-intensity oral anticoagulation with warfarin and low-dose aspirin in the primary prevention of ischemic heart disease in men at increased risk. Lancet. 1998;351:233-41.

5. Sonnenberg FA, Beck JR. Markov models in medical decision making: a practical guide. Med Decis Making. 1993;13:322-38.

6. Anderson KM, Wilson PW, Odell PM, Kannel WB. An updated coronary risk profile. Circulation. 1991;83:356-62.

7. Steering Committee of the Physician's Health Study Research Group. Final report of the aspirin component of the ongoing Physician's Health Study. N Engl J Med. 1989;321:129-35.

8. Peto R, Gray R, Collins R, et al. Randomized trial of prophylactic daily aspirin in British male doctors. BMJ. 1988;296:313-6.

9. Wolf PA, D'Agostino RB, Belange AJ, Kennel WB. Probability of stroke: a risk profile from Framingham study. Stroke. 1991;22:312-8.

10. Manson JE, Stampfer MJ, Colditz GA, et al. A prospective study of aspirin use and primary prevention of cardiovascular disease in women. JAMA. 1991;266:521-7.

11. Wilson PW, Evans JC. Coronary artery disease prediction. Am J Hypertens. 1993;6:S309-13.

12. Kannel WB, Larson M. Long-term epidemiologic prediction of coronary disease: the Framingham experience. Cardiology. 1993;82: 137-52.

13. Leaverton PE, Sorlie PD, Kleinman JC, et al. Representativeness of the Framingham risk model for coronary heart disease mortality: a comparison with a national cohort study. J Chron Dis. 1987;40: 775-84.

14. The Salt Collaborative Group. Swedish Aspirin Low-Dose Trial (SALT) of $75 \mathrm{mg}$ aspirin as secondary prophylaxis after cerebrovascular ischaemic events. Lancet. 1991;338:1345-9.

15. Giovanucci E, Rim EB, Stampfer MS, Colditz GA, Aschero A, Willer WC. Aspirin use and the risk for colorectal cancer and adenoma in male health professionals. Ann Intern Med. 1994;121:241-6.

16. Orencia A, Bailey K, Yawn BP, Kottke TE. Effect of gender on long term outcome of angina pectoris and myocardial infarction/sudden unexpected death. JAMA. 1993;269:2392-7.

17. Beck JR. Technical note. Med Decis Making. 1989;9:150.

18. Weinstein MC, Coxson PG, Williams LW, Pass TM, Stason WB, Goldman L. Forecasting coronary disease incidence, mortality, and cost: the coronary heart disease policy model. Am J Public Health. 1987;77:1417-26.

19. Stevenson R, Ranjadayalan K, Wilkinson P, Roberts R, Timmis A. Short and long term prognosis of acute myocardial infarction since the introduction of thrombolysis. BMJ. 1993;307:349-53.

20. Wolf PA, D’Agostino EB, O'Neal A, et al. Secular trends in stroke incidence and mortality: the Framingham study. Stroke. 1992;23: 1551-5.

21. McGovern PG, Burke GL, Sprafka JM, Xue S, Folsom AR, Blackburn H. Trends in mortality, morbidity, and risk factor levels for 
stroke from 1960 through 1990: the Minnesota heart survey. JAMA. 1992;268:753-9.

22. Howard G, Toole JF, Becker C, et al. Changes in survival following stroke in five North Carolina counties observed during two different periods. Stroke. 1989;20:345-50.

23. Dennis MS, Burn JP, Sandercock PA, Bamford JM, Wade DT, Warlow CP. Long term survival after first-ever stroke: the Oxfordshire Community Stroke Project. Stroke. 1993;24:796-800.

24. Sacco RL, Shi T, Zamanillo MC, Kargman DE. Predictors of mortality and recurrence after hospitalized cerebral infarction in an urban community: the Northern Manhattan Stroke Study. Neurology. 1994;44:626-34.

25. Department of Health and Human Services. Vital Statistics of the United States, 1988. Hyattsville, Md: National Center for Health Statistics; 1992.

26. Naglie IG, Detsky AS. Treatment of chronic nonvalvular atrial fibrillation in the elderly: a decision analysis. Med Decis Making. 1992;12:239-49.

27. Gage BF, Cardinalli AB, Albers GW, Owens DK. Cost-effectiveness of warfarin and aspirin for prophylaxis of stroke in patients with nonvalvular atrial fibrillation. JAMA. 1995;274:1839-45.
28. Tsevat J, Goldman L, Soukup JR, et al. Stability of time-tradeoff utilities in survivors of myocardial infarction. Med Decis Making. 1993;13:161-5.

29. Gabriel SE, Campion EM, Fallon WM. Patient preferences for nonsteroidal antiinflammatory drug related gastrointestinal complications and their prophylaxis. J Rheumatol. 1993;20:358-61.

30. Canadian Task Force on the Periodic Health Examination. Periodic health examination, 1991 update, 6: actylsalicylic acid and the primary prevention of cardiovascular disease. Can Med Assoc J. 1991; 145:1091-5.

31. Fuster V, Dyken ML, Vokonas PS, Hennekens C. AHA Medical/ Scientific Statement: aspirin as a therapeutic agent in cardiovascular disease. Circulation. 1993;87:659-75.

32. Hirsh J, Dalen JE, Fuster V, Harker LB, Salzman EW. Aspirin and other platelet-active drugs: the relationship between dose, effectiveness and side effects. Chest. 1992;102:S327-36.

33. Salzmann P, Kerlikowske K, Phillips K. Cost-effectiveness of extending screening mamography guidelines to include women 40 to 49 years of age. Ann Intern Med. 1997;127:955-65.

34. Kassirer JP. Incorporating patients' preferences into medical decisions. N Engl J Med. 1994;330:1895-6.

\section{JOURNAL OF GENERAL INTERNAL MEDICINE SUBSCRIBERS Do we have your new address?}

Send us your new address three months before it becomes effective, so we will have time to get it into our computer system and ensure that your copies of JGIM continue to arrive uninterrupted. Send your old mailing label, your new address with zip code, the effective date of your new address, and your current telephone number.

\author{
Nonmember subscribers notify: \\ Tina Lynch \\ Blackwell Science, Inc. \\ Commerce Place, 350 Main St. \\ Malden, MA 02148
}

\author{
SGIM members notify: \\ Janice L. Clements \\ Society of General Internal Medicine \\ 2501 M Street, NW, Suite 575 \\ Washington, DC 20037
}

